Bundesgesundheitsbl - Gesundheitsforsch Gesundheitsschutz 2006 • 49:396-398

DOI 10.1007/s00103-006-1253-4

๑) Springer Medizin Verlag 2005
Mitteilungen des Arbeitskreises Blut des Bundesministeriums für Gesundheit

\section{Vorgehensweise bei Variante Creutzfeldt-Jakob-Krankheit (vCJK) im Zusammenhang mit Blut, Plasma und Blutprodukten}

\author{
Bei der 61. Sitzung des Arbeitskreises Blut am \\ 11.1.2006 wurde folgendes Votum (V33) verabschiedet:
}

D urch das nachfolgende Verfahren sollen Übertragungen von vCJK durch noch nicht angewendete Blutprodukte verhindert, weitere potenziell infektiöse Spenden vermieden und eventuell stattgefundene Übertragungen aufgeklärt werden.

Das Verfahren gilt, wenn

a) bei einem Spender von Blut, Plasma oder anderen Bestandteilen aus Blut eine vCJK wahrscheinlich ist oder gesichert wurde,

b) bei einem Empfänger von Blut, Plasma oder Blutprodukten eine vCJK wahrscheinlich ist oder gesichert wurde.

\section{Feststellung einer vCJK}

Wird bei einer Person anhand der Klassifikationskriterien der WHO (siehe Anlage 1) eine wahrscheinliche oder gesicherte vCJK festgestellt, so wird vom behandelnden Arzt ermittelt, ob diese Person Blut, Plasma oder andere Komponenten aus Blut gespendet (Spender) oder Blut oder Blutprodukte erhalten hat (Empfänger).

In beiden Fällen sollte die Erkrankung durch ein ausgewiesenes Zentrum für spongiforme Enzephalopathien bestätigt worden sein. Gemäß $₫ 6$ Infektionsschutzgesetz muss der Krankheitsverdacht, die Erkrankung und der Tod an vCJK namentlich an das Gesundheitsamt gemeldet werden.

\section{Sofortmaßnahmen und Einleitung eines Rückverfolgungs- verfahrens}

\subsection{Vom Spender ausgehende Sofortmaßnahmen und Rückverfolgung}

Ergibt sich bei einem Spender eine wahrscheinliche oder gesicherte vCJK, wird folgendes Verfahren eingeleitet:

2.1.1 Es werden unverzüglich noch vorhandene(s) Blut, Plasma und weitere Blutkomponenten durch die Spendeeinrichtung gesperrt und für weitere Untersuchungen asserviert.

2.1.2 Es werden durch die Spendeeinrichtung alle Spenden von diesem Spender und die daraus gewonnenen Komponenten bzw. Bestandteile aus Blut rückwirkend bis maximal 30 Jahre vor der Diagnosestellung ermittelt.

2.1.3 Es werden die Einrichtungen der Krankenversorgung, an die die ermittelten Blutkomponenten geliefert wurden, durch die Spendeeinrichtung unverzüglich benachrichtigt. Noch in der Laufzeit befindliche Blutkomponenten werden zurückgerufen und asserviert. Über den Vollzug wird die Spendeeinrichtung informiert.

2.1.4 Die nach dem Qualitätssicherungssystem beauftragte ärztliche Person der
Einrichtung der Krankenversorgung soll grundsätzlich die Information und Aufklärung betroffener Empfänger von Blutkomponenten sicherstellen, vorzugsweise durch den behandelnden Arzt (z.B. Arzt der Klinik, niedergelassenen Facharzt, Hausarzt). Die betroffenen Empfänger sollen zeitnah über die mögliche Exposition und das damit verbundene mögliche Übertragungsrisiko informiert werden, verbunden mit der Empfehlung, künftig kein(e) Blut, Organe oder andere Gewebe zu spenden (siehe Muster in Anlagen 2 und 3 ). Die Einbeziehung eines ausgewiesenen Zentrums für spongiforme Enzephalopathien in die Aufklärungsmaßnahmen wird empfohlen (siehe z.B. Adressen unten). Erfolgt in begründeten Fällen keine Aufklärung des Patienten, so ist dies zu dokumentieren. Erfolgt die Information des Patienten durch den behandelnden Arzt, ergeht eine Rückmeldung an den Spendedienst darüber, ob er informiert werden konnte.

2.1.5 Die Spendeeinrichtung informiert parallel und unverzüglich pharmazeutische Unternehmer, die Blut, Plasma oder weitere Bestandteile aus Blut von dem betroffenen Spender erhalten haben.

2.1.6 Pharmazeutische Unternehmer, die Blut, Plasma und weitere Bestandteile aus Blut zur weiteren Verarbeitung erhalten haben und daraus Arzneimittel hergestellt und/oder in den Verkehr gebracht haben, 


\section{Anlage 1 \\ Diagnostische Kriterien der Variante der Creutzfeldt-Jakob-Krankheit (vCJK). Modifiziert nach WHO (2003)}

\begin{tabular}{ll}
\hline I & A Progrediente neurospsychiatrische Erkrankung \\
& B Erkrankungsdauer $>6$ Monate \\
C Routineuntersuchung lässt keine alternative Diagnose zu & D keine bekannte iatrogene Exposition \\
& E keine familiäre Prionerkrankung \\
& A Frühe psychiatrische Symptome \\
& B persistierende schmerzhafte Par-/Dysästhesien \\
II & C Ataxie \\
& D Demenz \\
& E Myoklonien oder Chorea oder Dystonie \\
& A EEG zeigt keine typischen Zeichen einer sporadischen CJK \\
III & (oder es wurde kein EEG abgeleitet) \\
& B im MRT hyperintenses Signal im Thalamus ("pulvinar sign") \\
IV & A PrP ${ }^{\text {Sc }}$ in lymphatischem Gewebe, z.B. Tonsillenbiopsie \\
Diagnose & I A und neuropathologische Bestätigung von vCJK \\
gesichert: & \\
Diagnose & I A und 4/5 von II und III A und III B \\
wahrscheinlich: & oder I A-E und IV A ${ }^{\mathrm{c}}$ \\
Diagnose & I A-E und 4/5 von II und III A \\
möglich: &
\end{tabular}

\footnotetext{
${ }^{\mathrm{a}}$ Depression, Angstzustände, Apathie, Wahnvorstellungen, Verlangsamung,

${ }^{\mathrm{b}}$ generalisierte, periodisch auftretende, bi-und triphasische Komplexe (0,5-2 pro Sekunde),

${ }^{c}$ Tonsillenbiopsie wird nicht routinemäßig empfohlen, auch nicht in Fällen mit für sporadische CJK typischem EEG. Eine Biopsie kann aber im klinischen Verdachtsfall einer vCJK ohne "pulvinar sign" im MRT hilfreich sein.

${ }^{\mathrm{d}}$ Spongiforme Veränderungen und Ablagerung von $\mathrm{PrP}^{\mathrm{Sc}}$ mit floriden Plaques im gesamten Cerebrum und Cerebellum
}

sperren die involvierten Produkte und veranlassen den Rückruf. Bei der Weitergabe von Zwischenprodukten muss eine Information an den Abnehmer erfolgen, der die betroffenen Zwischenprodukte und daraus hergestellte Produkte ebenfalls sperrt und zurückruft.

2.1.7 Es ergeht durch die Spendeeinrichtung unverzüglich eine Meldung an die nach Arzneimittelgesetz (AMG) und Transfusionsgesetz (TFG) zuständigen Landesbehörden und die Bundesoberbehörde.

2.1.8 Die pharmazeutischen Unternehmer, die Produkte aus Plasma und weiteren Bestandteilen aus Blut hergestellt und/ oder in den Verkehr gebracht haben, nehmen zusammen mit den nach AMG und TFG zuständigen Behörden des Bundes und der Länder eine Risikobewertung vor und empfehlen in Abhängigkeit von dieser ggf. weitere Maßnahmen.
2.1.9 Die entsprechend 2.1.6 betroffenen Empfänger von Blutprodukten werden grundsätzlich unter Berücksichtigung der Risikobewertung des pharmazeutischen Unternehmers und der zuständigen Behörden ebenfalls entsprechend 2.1.4 durch einen Arzt informiert und aufgeklärt.

2.1.10 Nach Abschluss des Verfahrens erstellen der/die pharmazeutischen Unternehmer einen Bericht und übermitteln diesen an die nach AMG und TFG zuständigen Landesbehörden und an die Bundesoberbehörde.

2.1.11 Verstirbt ein Empfänger, der Blutprodukte von einem an vCJK Erkrankten oder wahrscheinlich Erkrankten bekommen hat, so ist eine Autopsie unabhängig von der Todesursache anzustreben, um Untersuchungen auf vCJK durchzuführen.

\subsection{Vom Empfänger ausgehende Sofortmaßnahmen und Rückverfolgung}

Ist bei einem Patienten eine vCJK wahrscheinlich oder gesichert, so werden von der behandelnden ärztlichen Person folgende Schritte eingeleitet:

2.2.1 Es wird ermittelt, ob, wann und welche Blutprodukte der Patient erhalten hat.

2.2.2 Hat der Patient vor Beginn der vCJK-Symptomatik Blutprodukte erhalten (Empfänger), ergeht eine Meldung des Verdachts einer Arzneimittelnebenwirkung an das Paul-Ehrlich-Institut als zuständige Bundesoberbehörde und die zuständigen Landesbehörden. Die Meldungen an die Arzneimittelkommission der deutschen Ärzteschaft bleiben unberührt.

2.2.3 Die Bundesoberbehörde und die zuständigen Landesbehörden erfassen jeden einzelnen Fall und nehmen eine Bewertung vor, aus der sich ggf. weitere Maßnahmen ergeben.

2.2.4 Es werden die pharmazeutischen Unternehmer vom Paul-Ehrlich-Institut über die Möglichkeit einer Übertragung von vCJK durch die ermittelten Blutkomponenten und anderen Blutprodukte informiert.

2.2.5 Involvierte Spendeeinrichtungen und Blutkomponentenhersteller ermitteln die betroffenen Spender und dokumentieren den Vorgang. Soweit noch vorhanden, erfolgt dies in den Spenderakten. Gemäß $\$ 11$ Transfusionsgesetz sind die Angaben zur Spendenentnahme mindestens 30 Jahre aufzubewahren, wenn diese für die Rückverfolgung benötigt werden.

2.2.6 Eine Spenderinformation erfolgt nur dann, wenn die Spenden des betroffenen Spenders in weitere vCJK-Erkrankungsfälle involviert sind.

2.2.7 Der pharmazeutische Unternehmer, der involvierte Produkte aus Blut und Blutbestandteilen hergestellt und/oder in den Verkehr gebracht hat, dokumentiert die betroffenen Chargen, um im Falle weiterer 
vCJK-Fälle bei anderen Empfängern oder Spendern ggf. eine Verknüpfung feststellen zu können.

\section{Weitere Informationen können Sie unter folgenden (Internet) Adressen erhalten:}

\section{Nationales Referenzzentrum für die Surveillance humaner spongiformer Enzephalopathien,}

Universitätsklinikum Göttingen, Robert Koch Str. 40, 37075 Göttingen,

Tel. 0551-39-6636 oder 39-8401, E-Mail: epicjd@med.uni-goettingen.de, Homepage: www.cjd-goettingen.de

\section{Nationales Referenzzentrum für die Surveillance humaner spongiformer Enzephalopathien, Zentrum für Neuropathologie und Prionforschung, LMU München,}

Feodor-Lynen Str. 23,

81377 München,

Tel. o89-2180-780oo,

Homepage: www.znp-muenchen.de

\section{Robert Koch-Institut, \\ Arbeitskreis Blut,}

Nordufer 20, 13353 Berlin,

Tel. o1888-75440,

Homepage: www.rki.de

\section{Universität Edinburgh, National Creutzfeldt-Jakob Disease} Surveillance Unit,

Homepage: www.cjd.ed.ac.uk

(Informationen auf Englisch)

\section{Anlagen}

Anlage 1: Diagnostische Kriterien der Variante Creutzfeldt-Jakob-Krankheit (vCJK)

Anlage 2: Musterformulierungen zur Benachrichtigung des Arztes und zur Orientierung für die individuelle ärztliche Aufklärung bei nachträglich festgestelltem Expositionsverdacht, nachträglich festgestellter Exposition oder Infektion von Patienten mit vCJK durch Blut und Blutprodukte von Spendern, die nach der Spende an vCJK erkrankt sind.
Anlage 3: Liste von möglichen Fragen und Antworten für Ärzte zum Gespräch mit betroffenen Patienten.

Die Anlagen 2 und 3 können bei Bedarf bei der Geschäftsstelle des Arbeitskreises Blut angefordert werden.

Für den Arbeitskreis Blut: Prof. Dr. R. Burger, Vorsitzender; Dr. R. Offergeld, Geschäftsführerin

Eine englische Fassung dieses Votums ist verfügbar unter www.rki.de $>$ English $>$ Prevention of infection $>$ National advisory committee "Blood" 